\title{
Discovery of natural inhibitors targeting 2 - trans enoyl acyl carrier protein reductase in Mycobacterium tuberculosis by structure based drug designing
}

Saurov Mahanta ${ }^{1}$, Purvita Chowdhury ${ }^{1}$, Shamsun Nahar ${ }^{2}$, Bhaben Tanti ${ }^{2}$, P.J. Handique ${ }^{3, *}$

${ }^{1}$ National Institute of Electronics and Information Technology, Guwahati-781008, Assam, India.

2Department of Botany, Gauhati University, Guwahati 781014, Assam, India.

3Department of Biotechnology, Gauhati University, Guwahati 781014, Assam, India.

Received: July 27, 2016; Revised: August 11, 2016; Accepted: August 23, 2016

\begin{abstract}
Evolution and the rapid spread of the multidrug resistant Mycobacterium tuberculosis (Mtb) have posed a serious crisis. Moreover, the available first line drugs also confer adverse effects on the patients suffering from tuberculosis (TB) thus making the cure increasingly difficult. Thus the search of novel and potent natural compounds targeting anti-tubercular agents has become inevitable. Here, we report identification of potential natural anti-tubercular candidates targeting Mtb 2-Trans Enoyl Acyl Carrier Protein Reductase (InhA) of the fatty acid pathway using structure based drug designing. In the present study, we selected a total of 154 compounds from three plants i.e. Ginkgo biloba, Neem (Azadirachta indica) and Tea (Camellia sinensis) which were obtained from PubChem Compounds. These compounds were subjected to Lipinski's rule of five and drug likeness filters. Finally, the compounds were docked at the active site of Mtb InhA (PDB code: 3FNE) using AutoDock Vina to select inhibitors with favourable interactions. The structure based ligand receptor docking aided in the identification of a number of natural candidates which had high binding affinities against Mtb InhA. Thus, these molecules could potentially inhibit Mtb InhA and succor to the development of lead compounds in the experimental drug discovery of anti-tuberculars.
\end{abstract}

Key words: InhA; natural compounds; docking; Mycobacterium tuberculosis (Mtb); drug designing

\section{Introduction}

Mycobacterium tuberculosis (Mtb), the causative agent of tuberculosis (TB) is one of the major health threats especially in Asia and Africa. In 2012, an estimated 8.6 million people developed TB and 1.3 million died from the disease (Anonymous, 2013). Moreover, the vicious interactions between the human immunodeficiency virus (HIV) with $\mathrm{TB}$ and emergence of new multi-drug resistant tuberculosis (MDR-TB) have created serious challenges in the control of TB. The current TB control measures include over 20 existing drugs of which isoniazid, ethambutol, pyrazinamide and rifampin are used as front-line drugs (Raman et al., 2008). But these front line drugs also have several shortcomings like high levels of adverse effects and drug inactivation by the emerging drug-resistant strains (Nunn et al., 2005). Thus, new drugs are necessary to inhibit the pathogen by targeting its metabolic pathways which are essential for the pathogen to survive.

One of the current effective targets for anti TB drugs is the cell wall biosynthesis. The $i n h A$ gene is involved in the production of very-long-chain fatty acids known as mycolic acids, which are one of the major components of the Mycobacterium cell wall formation (Banerjee et al., 1994). InhA (Rv1484) encodes an $\mathrm{NADH}$-dependent 2-trans-enoyl-ACP reductase which catalyzes the elongation of FAS-II leading to the reduction of 2-trans-enoyl chains (_C12) to obtain the saturated chains (Dessen et al., 1995; Quemard et al., 1995). The gene $i n h A$ has $25 \%$ sequence identity to a gene encoding a human protein (pecR) and $31 \%$ to a mouse protein (Decr1) making it a "host-safe" target
(Hasan et al., 2006). Drugs like isoniazid and broad spectrum antibacterial Triclosan inhibits the biosynthesis of mycolic acids (Schroeder et al., 2002). However clinical strains have been isolated which are resistant to isoniazid (Vilchèze et al., 2006).

The screening of natural products provides greater structural diversity than standard synthetic chemistry and offers significant opportunities for finding novel low molecular weight lead compounds. Moreover, it is a known fact that natural products are the basis of formulations of new synthetic compounds. In traditional medicine, tea (Camellia sinensis), neem (Azadiracbta indica) and Ginkgo biloba (Ginkgo biloba) have been used for treatment of various infections. It has been reported that they exhibit anti bacterial, antifungal, anti pesticidal and even anti cancerous properties (Wang and Ng, 2000; Sharma, 2006; Gbotolorun et al., 2008; Schumacher et al., 2011). Recently tea flavonoids have been accounted as antituberculosis agents (Lin et al., 2002; Lim et al., 2001). As per our knowledge the above mentioned natural products have not yet been screened against the $M t b$ InhA target. Considering the importance of $\operatorname{Inh} A$ in $M t b$ survival and constantly evolving MDR-TB strains, we have attempted to screen natural compounds as $M t b$ InhA inhibitors using structure based computer aided drug designing (CADD) method. This study would aid the translational studies and further help in developing novel drugs against $M t b$.

\section{*Corresponding Author:}

Dr. P. J. Handique,

Professor, Department of Biotechnology,

Gauhati University,

Guwahati 781014, Assam, India.

E-mail: pjh@gauhati.ac.in

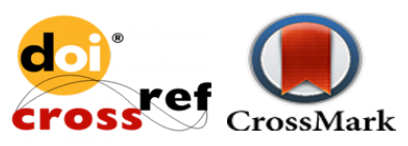




\section{Materials and Methods}

\section{Preparation of InhA structure}

The 3D structure of InhA complexed with 2-(2,4dichlorophenoxy)-5-(pyridine-2-ylmethyl) phenol and Nicotinamide adenine dinucleotide was obtained from the Protein Data Bank [PDB: 3FNE]. Inh $A$ consists of four chains namely $\mathrm{A}$ with 268 amino acids, B, C and D with 254 amino acids each while the active site residues are located at the chain A. The sequences of the individual chains were aligned using ClustalX. It was found that all the chains had hundred percent similarities and hence only one chain i.e. chain A was selected for the study as it contains the residues of the active site. For the structure-based molecular docking processes the InhA_A structure was prepared by removing all ligands along with the solvents, bound cofactors and by adding polar hydrogen. This was done using the extensible molecular modeling software UCSF Chimera (Pettersen et al., 2004) and energy was minimized using VegaZZ (Pedretti et al., 2004) software. Ten best ligand binding sites were predicted for the molecular docking using the online software Q-SiteFinder (Laurie and Jackson, 2005).

\section{Preparation of ligands}

The IUPAC name and the canonical SMILES of 83 tea polyphenols, 33 neem polyphenols and 38 Gingko biloba compounds were retrieved from PubChem compound (http://www.ncbi.nlm.nih.gov/pccompound). The $2 \mathrm{D}$ structures and molecular weights were generated using the software ChemDraw Ultra 8.0 (Cambridge Soft Corporation, MA 02140 U.S.A). 3D structures were subsequently obtained from Online Corina (Sadowski et al., 1994) with the help of SMILES. Drug likeness property was also checked by using online software Molsoft (http://molsoft.com/mprop/). The torsion angles of the ligands were modified by using TORSDOF utility of AutoDock tools and the pdbqt [a special file format generated by AutoDock tools] files of the ligands were generated.

\section{Molecular Docking}

AutoDock Vina was used for the Molecular docking (Trott and Olson, 2010), due to its high speed and accuracy as compared to its predecessor, AutoDock 4 (Morris et al., 2008). In order to prepare the input pdbqt file for InhA protein and also to set the center and the size of the grid box AutoDock Tools was utilized. The grid box cavity was set as $30 \times 30$ x 30 and centre was set at $\mathrm{x}=31.066, \mathrm{y}=0.295, \mathrm{z}$ $=19.543$ for the protein's active site. The scoring function of AutoDock Vina is used to calculate the predicted binding affinity $(\mathrm{kcal} / \mathrm{mol})$. This scoring function is divided into two parts: i) a conformation-dependent part which is the sum of intermolecular and intramolecular contributions, including hydrogen bonding, hydrophobic, and steric interactions ii) a conformation - independent part depending on the number of rotatable bonds between heavy atoms in the ligand. The scoring function AutoDock Vina gives a different weight to each of the interactions and number of rotatable bonds (Kakati et al., 2012; Trott and Olson, 2010). The docking was validated by redocking the InhA active ligands into their binding sites.

\section{Results and Discussion}

A structure based approach was taken in this study to find the potential inhibitors against the InhA protein of the $M t b$ from plant compounds. The coordinates of the grid box in molecular docking play a very important role in ensuring that the ligands bind in the correct conformation to the binding pocket. To validate this study, the cocrystallized ligands were again docked into their binding sites respectively with InhA_A chain of $M t b$ InhA protein using Autodock tools. The binding conformations were similar to the co-crystallized binding modes of the ligands with binding affinities as given in table 1 . The compounds from three different plants viz tea, neem, Gingko biloba were screened with the same coordinates of the grid box.

Table 1: Docking result of InhA with that of some known inhibitors of the protein

\begin{tabular}{clc}
\hline S.No. & \multicolumn{1}{c}{ Known inhibitor } & $\begin{array}{c}\text { Affinity } \\
\text { (kcal/mol) }\end{array}$ \\
\hline 1 & (3S)_1_cyclohexyl_5_oxo_N_phenylpyrrolidine_3_carboxamide & -6.4 \\
2 & (3S)_1_cyclohexyl_N_(3_5_dichlorophenyl)_5_oxopyrrolidine_3_carboxamide & -6.5 \\
3 & (3S)_N_(3_bromophenyl)_1_cyclohexyl_5_oxopyrrolidine_3_carboxamide & -5.5 \\
4 & (3S)_N_(3_chloro_2_methylphenyl)_1_cyclohexyl_ & -7.4 \\
5 & 5_oxopyrrolidine_3_carboxamide & -6.5 \\
6 & 2_(2_4_dichlorophenoxy)_5_(2_phenylethyl)phenol & -6.4 \\
7 & 2_(2_4_dichlorophenoxy)_5_(pyridin_2_ylmethyl)phenol & -5.6 \\
8 & 5_(cyclohexa_1_5_dien_1_ylmethyl)_2_(2_4_dichlorophenoxy)phenol & -6.0 \\
9 & 5_benzyl_2_(2_4_dichlorophenoxy)phenol & -4.6 \\
10 & 5_octyl_2_phenoxyphenol & -5.3 \\
11 & 5_pentyl_2_phenoxyphenol & -3.0 \\
12 & 5_pentyl_2_phenoxyphenol & -5.0 \\
13 & Ethionamid & -5.5 \\
14 & Noniazid & -7.0 \\
15 & N_(4_methylbenzoyl)_4_benzylpiperidine & -6.7 \\
16 & Triclosan & -6.4 \\
\hline
\end{tabular}


Table 2: Tea polyphenols docked with target protein InhA

\begin{tabular}{|c|c|c|}
\hline $\begin{array}{l}\text { Common names } \\
\text { of compounds }\end{array}$ & IUPAC name & $\begin{array}{l}\text { Binding affinity } \\
\text { (kcal/mol) }\end{array}$ \\
\hline epicatechin_gallate & $\begin{array}{l}\text { [(2R,3R)-2-(3,4-dihydroxyphenyl)-5,7-dihydroxy-3,4- } \\
\text { dihydro-2H-chromen-3-yl] 3,4,5-trihydroxybenzoate }\end{array}$ & -9.4 \\
\hline Phloridzin & $\begin{array}{l}\text { 1-[2,4-dihydroxy-6-[(2S,3R,4S,5S,6R)-3,4, } \\
\text { 5-trihydroxy-6-(hydroxymethyl)oxan-2-yl]oxyphenyl]-3- } \\
\text { (4-hydroxyphenyl) } \\
\text { propan-1-one }\end{array}$ & -8.7 \\
\hline $\begin{array}{l}\text { Epigallocatechin_g } \\
\text { allate }\end{array}$ & $\begin{array}{l}\text { (2R,3R)-2-(3,4,5-trihydroxyphenyl)-3,4-dihydro- } 2 \mathrm{H} \text { - } \\
\text { chromene-3,5,7-triol }\end{array}$ & -8.4 \\
\hline Quercetin & 2-(3,4-dihydroxyphenyl)-3,5,7-trihydroxychromen-4-one & -8.3 \\
\hline Apigenin & 5,7-dihydroxy-2-(4-hydroxyphenyl)chromen-4-one & -8.3 \\
\hline N/A & 3_phenylpyridine & -8.2 \\
\hline P_Rosolic_acid & $\begin{array}{l}\text { 4-[bis(4-hydroxyphenyl)methylidene]cyclohexa-2,5-dien- } \\
\text { 1-one }\end{array}$ & -8.1 \\
\hline Purpurogallin & 2,3,4,5-tetrahydroxybenzo[7]annulen-6-one & -7.8 \\
\hline Catechin & $\begin{array}{l}\text { (2R,3S)-2-(3,4-dihydroxyphenyl)-3,4-dihydro- } 2 \mathrm{H}- \\
\text { chromene-3,5,7-triol }\end{array}$ & -7.8 \\
\hline- & 3_propylquinoline & -7.8 \\
\hline- & $\begin{array}{l}\text { (E)_4_11_11_trimethyl_8_methylenebicyclo[7_2_0]unde } \\
\text { c_4_ene }\end{array}$ & -7.7 \\
\hline Resvertrol & 5-[(E)-2-(4-hydroxyphenyl)ethenyl]benzene-1,3-diol & -7.6 \\
\hline Morin & 2-(2,4-dihydroxyphenyl)-3,5,7-trihydroxychromen-4-one & -7.3 \\
\hline- & 2_4_dimethylquinoline & -7.3 \\
\hline Ellagic acid & $\overline{\mathrm{N}} / \overline{\mathrm{A}}$ & -7.2 \\
\hline Genistein & 5,7-dihydroxy-3-(4-hydroxyphenyl)chromen-4-one & -7.1 \\
\hline- & 2_methylquinoline & -6.9 \\
\hline Epicatechin & $\begin{array}{l}\text { (2R,3R)-2-(3,4-dihydroxyphenyl)-3,4-dihydro-2H- } \\
\text { chromene-3,5,7-triol }\end{array}$ & -6.8 \\
\hline myrecetin & $\begin{array}{l}\text { 3,5,7-trihydroxy-2-(3,4,5-trihydroxyphenyl)chromen-4- } \\
\text { one }\end{array}$ & -6.6 \\
\hline- & 2_isopropyl_5_methylphenol & -6.6 \\
\hline Emodin & 1,3,8-trihydroxy-6-methylanthracene-9,10-dione & -6.5 \\
\hline- & 3_3a_dihydroisobenzofuran_1 $(7 \mathrm{aH})$ _one & -6.5 \\
\hline- & 1H_indole & -6.5 \\
\hline- & 1_methyl_4_(prop_1_en_2_yl)cyclohex_1_ene & -6.5 \\
\hline Esculetin & 6,7-dihydroxychromen-2-one & -6.4 \\
\hline Chrysin & 5,7-dihydroxy-2-phenylchromen-4-one & -6.4 \\
\hline- & 3_7_7_trimethylbicyclo[4_1_0] hept_3_ene & -6.3 \\
\hline- & 2_methylbenzaldehyde & -6.2 \\
\hline Piceatannol & 5-[(E)-2-(3,4-dihydroxyphenyl)ethenyl]benzene-1,3-diol & -6.1 \\
\hline Phloretin & $\begin{array}{l}\text { 3-(4-hydroxyphenyl)-1-(2,4,6-trihydroxyphenyl)propan-1- } \\
\text { one }\end{array}$ & -6.1 \\
\hline $\begin{array}{l}\text { Nordihydroguaiar- } \\
\text { etic acid }\end{array}$ & $\begin{array}{l}\text { 4-[4-(3,4-dihydroxyphenyl)-2,3-dimethylbutyl]benzene- } \\
\text { 1,2-diol }\end{array}$ & -6.1 \\
\hline Baicalein & 5,6,7-trihydroxy-2-phenylchromen-4-one & -6.1 \\
\hline $\mathrm{N} / \mathrm{a}$ & 6_methylquinoline & -6.1 \\
\hline- & 4_8_dimethyl_quinoline & -6.1 \\
\hline- & 2_phenylethanol & -6 \\
\hline- & 5_ethyl_2_methylpyridine & -5.9 \\
\hline- & 2_5_dimethylpyridine & -5.9 \\
\hline- & (S_E $)$-3_7_11_trimethyldodeca_1_6_10_trien_3_ol & -5.9 \\
\hline- & 2_6_6_trimethylcyclohex_2_ene_1_4_dione & -5.7 \\
\hline- & 4_methoxybenzaldehyde & -5.6 \\
\hline- & 3_ethyl_2_5_dimethylpyrazine & -5.6 \\
\hline n_propylgallate & propyl 3,4,5-trihydroxybenzoate & -5.5 \\
\hline$-1 \quad-$ & 2_phenylpyridine & -5.5 \\
\hline- & 2_ethylpyridine & -5.5 \\
\hline- & 2_ethyl_3_5_dimethylpyrazine & -5.5 \\
\hline- & 2_(4_methylcyclohex_3_enyl)propan_2_ol & -5.4 \\
\hline Phloroglucinol & benzene-1,3,5-triol & -5.3 \\
\hline $\mathrm{N} / \mathrm{a}$ & 6_amino_5_methylpyrimidin_2 $(1 \mathrm{H})$ _one & -5.3 \\
\hline- & 4_vinylpyridine & -5.3 \\
\hline- & 1_(pyridin_2_yl)ethanone & -5.3 \\
\hline- & 4_methylpyridine & -5.1 \\
\hline- & 2_6_dimethylquinoline & -5.1 \\
\hline- & 3_methylpyridine & -5 \\
\hline- & 2_methylpyridine & -5 \\
\hline- & 2_ethylhexan_1_ol & -4.9 \\
\hline- & 1_(furan_2_yl)ethanone & -4.9 \\
\hline- & 3_methoxypyridine & -4.8 \\
\hline- & 1_(1H_pyrrol_2_yl)ethanone & -4.8 \\
\hline- & 2_methylbenzo[d] thiazole & -4.7 \\
\hline- & 2_6_6_trimethylcyclohex_2_enone & -4.7 \\
\hline- & (E)_4_methyl_2_phenylpent_2_enal & -4.7 \\
\hline- & 4_butylquinoline & -4.6 \\
\hline
\end{tabular}




\begin{tabular}{cll}
\hline- & 2_ethyl_6_methylpyridine & -4.6 \\
- & 2_ethyl_6_methylpyrazine & -4.6 \\
- & 2_5_dimethylthiazole & -4.5 \\
- & 4_methyl_2_phenyl_pent_2_en_1_al & -4.4 \\
- & 2_ethyl_5_methylpyrazine & -4.3 \\
- & 3_butylpyridine & -4.2 \\
- & S_E)_3_7_dimethylocta_1_5_7_trien_3_ol & -4.2 \\
- & 4_ethyl_2_methoxyphenol & -4.1 \\
- & 2_6_10_14_tetramethylpentadecane & -4.1 \\
- & 5_isopropylheptan_2_one & -4 \\
- & 2_4_dimethylthiazole & -4 \\
- & (S_E)_2_6_dimethylocta_3_7_diene_2_6_diol & -3.9 \\
- & 4_ethyl_2_5_dimethylthiazole & -3.8 \\
- & 2_5_dimethylpyrazine & -3.8 \\
- & 2_4_5_trimethylthiazole & -3.8 \\
- & 5_methylthiazole & -3.7 \\
- & 2_6_dimethylpyrazine & -3.7 \\
- & 6_10_dimethylundecan_2_one & -3.5 \\
- & 2_phenylacetaldehyde & -3.5 \\
\hline
\end{tabular}

Table 3: Neem compounds docked with the InhA protein

\begin{tabular}{|c|c|c|}
\hline $\begin{array}{l}\text { Common names of } \\
\text { compounds }\end{array}$ & IUPAC name & $\begin{array}{c}\text { Binding affinity } \\
(\mathrm{kcal} / \mathrm{mol})\end{array}$ \\
\hline Azadirone & $\begin{array}{l}\text { [(5R,7R,9R,10R,13S,17R)-17-(furan-3-yl)-4,4,8,10,13- } \\
\text { pentamethyl-3-oxo-5, } \\
6,7,9,11,12,16,17 \text {-octahydrocyclopenta[a]phenanthren-7-yl] } \\
\text { acetate }\end{array}$ & -8.4 \\
\hline Nimocinolide & ( & -8 \\
\hline Kulactone & - & -7.9 \\
\hline Dianabol & $\begin{array}{l}\text { (8R,9S,10R,13S,14S,17S)-17-hydroxy-10,13,17-trimethyl- } \\
\text { 7,8,9,11,12,14,15, } \\
\text { 16-octahydro-6H-cyclopenta[a]phenanthren-3-one }\end{array}$ & -7.8 \\
\hline Gedunin & - & -7.6 \\
\hline Nimbin & - & -7.4 \\
\hline Emetine & $\begin{array}{l}\text { (2S,3R,11bS)-2-[[(1R)-6,7-dimethoxy-1,2,3, } \\
\text { 4-tetrahydroisoquinolin-1-yl]methyl]-3-ethyl-9,10- } \\
\text { dimethoxy-2,3,4,6,7, } \\
\text { 11b-hexahydro-1H-benzo[a]quinolizine }\end{array}$ & -7.4 \\
\hline Quinine & $\begin{array}{l}\text { 5R)-5-ethenyl-1-azabicyclo[2.2.2] octan-2-yl]-(6- } \\
\text { methoxyquinolin-4-yl) } \\
\text { methanol }\end{array}$ & -7.3 \\
\hline Melianotriol & $\begin{array}{l}\text { (1S)-1-[(2R,4S,5R)-5-hydroxy-4-[(3S,9R,10R,13S,14S,17R)- } \\
\text { 3-hydroxy-4,4,10,13,14-pentamethyl- } \\
\text { 2,3,5,6,9,11,12,15,16,17-decahydro-1H- } \\
\text { cyclopenta[a]phenanthren-17-yl]oxolan-2-yl]-2- } \\
\text { methylpropane-1,2-diol }\end{array}$ & -7.1 \\
\hline Quercetin & 2-(3,4-dihydroxyphenyl)-3,5,7-trihydroxychromen-4-one & -7.1 \\
\hline Nimbiol & $\begin{array}{l}\text { 6-hydroxy-1,1,4a,7-tetramethyl-3,4,10, } \\
\text { 10a-tetrahydro-2H-phenanthren-9-one } \\
\text { (4aS,10aS)-6-hydroxy-1,1,4a-trimethyl-7-propan-2-yl- }\end{array}$ & -7 \\
\hline Sugiol & $\begin{array}{l}3,4,10 \\
10 a-t e t r a h y d r o-2 H-p h e n a n t h r e n-9-o n e\end{array}$ & -6.9 \\
\hline Velutin & $\begin{array}{l}\text { 5-hydroxy-2-(4-hydroxy-3-methoxyphenyl)-7- } \\
\text { methoxychromen-4-one } \\
\text { (4R,4aR,7S,7aR,12bS)-3-methyl-2,4,4a,7,7a,13-hexahydro- }\end{array}$ & -6.7 \\
\hline Morphine & $\begin{array}{l}\text { 1H-4, } \\
\text { 12-methanobenzofuro[3,2-e]isoquinoline-7,9-diol }\end{array}$ & -6.5 \\
\hline Stearic acid & Octadecanoic acid & -6.4 \\
\hline Copaene & - & -6.4 \\
\hline Eucalyptol & 2,2,4-trimethyl-3-oxabicyclo[2.2.2]octane & -6.2 \\
\hline Isocaryophyllene & $\begin{array}{l}\text { 1R,4Z,9S)-4,11,11-trimethyl-8- } \\
\text { methylidenebicyclo[7.2.0]undec-4-ene }\end{array}$ & -6.1 \\
\hline Germacrene acid & $\begin{array}{l}\text { 2-[(1R,3E,7E)-4,8-dimethylcyclodeca-3,7-dien-1-yl]prop-2- } \\
\text { enoate }\end{array}$ & -6 \\
\hline beta_ocimene & (3E)-3,7-dimethylocta-1,3,6-triene & -5.9 \\
\hline Cumarin & Chromen-2-one & -5.5 \\
\hline Dihydrochalcone & 1,3-diphenylpropan-1-one & -5.1 \\
\hline Phenylephrine & 3-[(1R)-1-hydroxy-2-(methylamino)ethyl]phenol & -5 \\
\hline 1_hexanol & hexan-1-ol & -4.6 \\
\hline nPentacosane & - & -3.8 \\
\hline Oleic acid & (Z)-octadec-9-enoic acid & -3.4 \\
\hline Dodecanol & Dodecan-1-ol & -3.4 \\
\hline
\end{tabular}




\begin{tabular}{lll}
\hline $\begin{array}{l}\text { Methyldithio_1_prop } \\
\text { ene }\end{array}$ & 1-(methyldisulfanyl)prop-1-ene & -3.3 \\
$11,14,17-$ & $(11 Z, 14 Z, 17 Z)$-icosa-11,14,17-trienoic acid & -3.3 \\
Eicosatrienoic acid & (9Z,12Z)-octadeca-9,12-dienoic acid & -3.1 \\
Linoleic acid & Hexadecanoic acid & -3 \\
\hline Palmitic acid &
\end{tabular}

The database of plant compounds were initially subjected to the Lipinski's rule of five for drug like compounds (Lipinski, 2000). Considering the condition of Lipinski's rule of five for a drug candidate, compounds having molecular weight less than $500 \mathrm{D}$ were chosen for performing proteinligand docking. A total of 154 compounds (83 compounds from tea, 33 compounds from neem and 38 compounds from Gingko biloba) were selected for the same. The binding affinity of the selected tea, neem and gingkobiloba compounds to the $M t b$ InhA protein is given in Table $3-5$ respectively. The best five molecules from each plant, based on their binding affinity to the receptor, were selected for the further study. The hydrogen bonding interaction between the receptor and the ligand was also analyzed using PyMol molecular viewer (http://www.pymol.org). However, among these best binding molecules, tea compounds viz. epicatchin_gallate and phloridzin showed 7 H-bond donors each and epigallocatechin_gallate showed $6 \mathrm{H}$-bond donors. Thus these molecules were not included further in the study. Similarly, two neem compounds viz. azadirone and kulactone had to be excluded from the study as the lipophilicity $(\log \mathrm{P})$ values were found to be higher than 5 .

Table 4: Gingko biloba compounds docked with the InhA protein

\begin{tabular}{|c|c|c|}
\hline $\begin{array}{l}\text { Common names of } \\
\text { compounds }\end{array}$ & IUPAC name & $\begin{array}{l}\text { Binding affinity } \\
\text { (Kcal/Mol) }\end{array}$ \\
\hline Tricetin & $\begin{array}{l}\text { 5,7-dihydroxy-2-(3,4,5-trihydroxyphenyl)chromen-4- } \\
\text { one }\end{array}$ & -8.8 \\
\hline Luteolin & 2-(3,4-dihydroxyphenyl)-5,7-dihydroxychromen-4-one & -8.6 \\
\hline Kaempferol & 3,5,7-trihydroxy-2-(4-hydroxyphenyl)chromen-4-one & -8.5 \\
\hline Quercetin & $\begin{array}{l}\text { 2-(3,4-dihydroxyphenyl)-3,5,7-trihydroxychromen-4- } \\
\text { one }\end{array}$ & -8.2 \\
\hline Catechin & $\begin{array}{l}\text { (2R,3S)-2-(3,4-dihydroxyphenyl)-3,4-dihydro-2H- } \\
\text { chromene-3,5,7-triol }\end{array}$ & -8 \\
\hline Ginkgolide A & $\mathrm{N} / \mathrm{A}$ & -7.9 \\
\hline Ginkgolide B & $\mathrm{N} / \mathrm{A}$ & -7.5 \\
\hline Ginkgolide C & $\mathrm{N} / \mathrm{A}$ & -7.4 \\
\hline Ginkgolide J & $\mathrm{N} / \mathrm{A}$ & -7.2 \\
\hline Ginkgolide M & $\mathrm{N} / \mathrm{A}$ & -7.2 \\
\hline Xanthurenic Acid & $\begin{array}{l}\text { 8-hydroxy-4-oxo-1H-quinoline-2-carboxylic acid } \\
\text { 17-(5-ethyl-6-methylhept-3-en-2-yl)-10,13-dimethyl- } \\
\text { 2,3,4,7,8,9,11,12,14, }\end{array}$ & -7.2 \\
\hline Stigmasterol & $\begin{array}{l}\text { 15,16,17-dodecahydro-1H-cyclopenta[a]phenanthren-3- } \\
\text { ol }\end{array}$ & -6.9 \\
\hline Bilobalide & $\mathrm{N} / \mathrm{A}$ & -6.7 \\
\hline Protocatechuic Acid & 3,4-dihydroxybenzoic acid & -6.5 \\
\hline Amygdalin & $\begin{array}{l}\text { 2-phenyl-2-[(2R,3R,4S,5S,6R)-3,4,5-trihydroxy-6- } \\
{[[(2 \mathrm{R}, 3 \mathrm{R}, 4 \mathrm{~S}, 5 \mathrm{~S}, 6 \mathrm{R})-3,4,5 \text {-trihydroxy-6- }} \\
\text { (hydroxymethyl)oxan-2-yl] oxymethyl]oxan-2-yl] } \\
\text { oxyacetonitrile }\end{array}$ & -6.1 \\
\hline Isorhamnetin & $\begin{array}{l}\text { 3,5,7-trihydroxy-2-(4-hydroxy-3- } \\
\text { methoxyphenyl)chromen-4-one }\end{array}$ & -6 \\
\hline Kynurenic Acid & $\begin{array}{l}\text { 4-oxo-1H-quinoline-2-carboxylic acid } \\
\text { 17-(5-ethyl-6-methylheptan-2-yl)- }\end{array}$ & -5.9 \\
\hline Sitosterol & $\begin{array}{l}\text { 2,3,4,7,8,9,10,11,12,13,14,15,16,17-tetradecahydro- } \\
\text { 10,13-dimethyl-1H-cyclopenta[a]phenanthren-3-ol }\end{array}$ & -5.8 \\
\hline Shikimic Acid & $\begin{array}{l}\text { (3R,4S,5R)-3,4,5-trihydroxycyclohexene-1-carboxylic } \\
\text { acid }\end{array}$ & -5.7 \\
\hline 4_Coumaric Acid & (E)-3-(4-hydroxyphenyl)prop-2-enoic acid & -5.6 \\
\hline Hexanoic Acid & hexanoic acid & -5.2 \\
\hline 4_Methoxymethylpyridoxine & $\begin{array}{l}\text { 5-(hydroxymethyl)-4-(methoxymethyl)-2-methylpyridin- } \\
\text { 3-ol }\end{array}$ & -4.9 \\
\hline Vanillic Acid & 4-hydroxy-3-methoxybenzoic acid & -4.6 \\
\hline Bilobol & 5-[(Z)-pentadec-8-enyl]benzene-1,3-diol & -4.6 \\
\hline Vitamin C & $\begin{array}{l}\text { (2R)-2-[(1S)-1,2-dihydroxyethyl]-3,4-dihydroxy-2H- } \\
\text { furan-5-one }\end{array}$ & -4.2 \\
\hline Citramalate & 2-hydroxy-2-methylbutanedioic acid & -4.1 \\
\hline Butyric Acid & Butyric Acid & -4.1 \\
\hline Anacardic Acid & 2-hydroxy-6-pentadecylbenzoic acid & -3.9 \\
\hline Cardanol & 3-[(8Z,11Z)-pentadeca-8,11,14-trienyl]phenol & -3.6 \\
\hline Ginkgolic Acid & 2-hydroxy-6-[(Z)-pentadec-8-enyl]benzoic acid & -3.4 \\
\hline
\end{tabular}


The drug likeness property was calculated for the remaining 10 best binding molecules using MolSoft program. The overall drug likeness is predicted by Drug likeness score using MolSoft's fingerprints and Support Vector Machine (SVM) classifier (Lalitha and Sivakamasundari, 2010). Drug likeness is a balance of diverse molecular properties of the compounds and positive score indicates that the compound has a probability of becoming a successful drug. Among all the remaining compounds, tricetin, a ginkgobiloba compound and gedunin, a neem compound showed a negative drug likeness score. After following the Lipinski's rule of five and checking the drug likeliness property, two tea polyphenols viz. quercetin and apigenin, two neem compound viz. dianabol and nimocinolide and four ginkgobiloba compounds viz. luteolin, kaempferol, quercetin and catechin fall within this barrier (Table 5).

Table 5: Top compounds chosen as lead compounds

\begin{tabular}{lcc}
\hline $\begin{array}{c}\text { Compound } \\
\text { name }\end{array}$ & $\begin{array}{c}\text { Binding affinity } \\
\text { (kcal/mol) }\end{array}$ & $\begin{array}{c}\text { Compound } \\
\text { category }\end{array}$ \\
\hline Luteolin & -8.6 & Ginkgobiloba \\
Kaempferol & -8.5 & Ginkgobiloba \\
Quercetin & -8.3 & Tea \\
Apigenin & -8.3 & Tea \\
Quercetin & -8.2 & Ginkgobiloba \\
Catechin & -8 & Ginkgobiloba \\
Dianabol & 7.8 & Neem \\
\hline
\end{tabular}

Further, the top 8 docking hits were analyzed in terms of their structures and ligand-receptor interactions. The interactions were found inside the binding cavity showing important interactions like hydrophobic interactions and hydrogen bonding. The tea polyphenol quercetin showed binding affinity of $-8.3 \mathrm{kcal} / \mathrm{mol}$ that formed two hydrogen bonds: one between $\mathrm{O}_{18}$ and $\mathrm{H}$ of Gly14 and another between $\mathrm{O}_{19}$ and $\mathrm{H}$ of $\mathrm{Val} 65$ (Fig. 1A). The tea polyphenol apigenin had a binding affinity of$8.3 \mathrm{kcal} / \mathrm{mol}$ and formed three hydrogen bonds: one between $\mathrm{O}_{20}$ and $\mathrm{H}$ of $\mathrm{Val} 65$ and the other two between $\mathrm{O}_{10}$, Ser94 and Gly14.

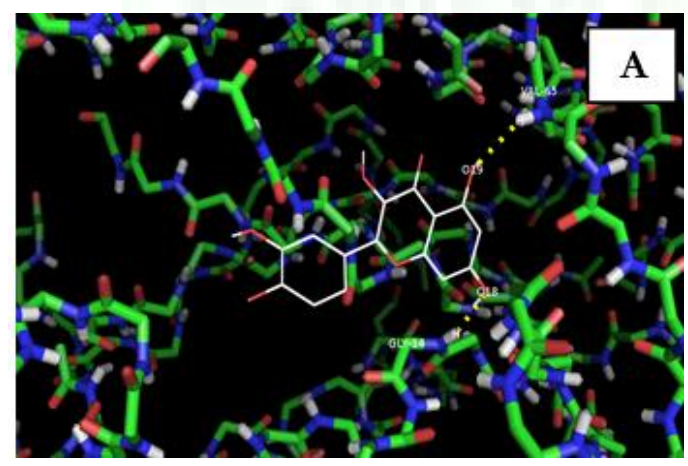

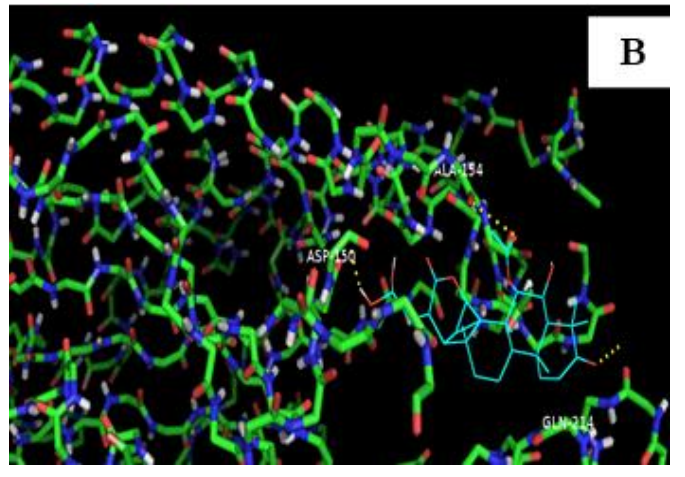

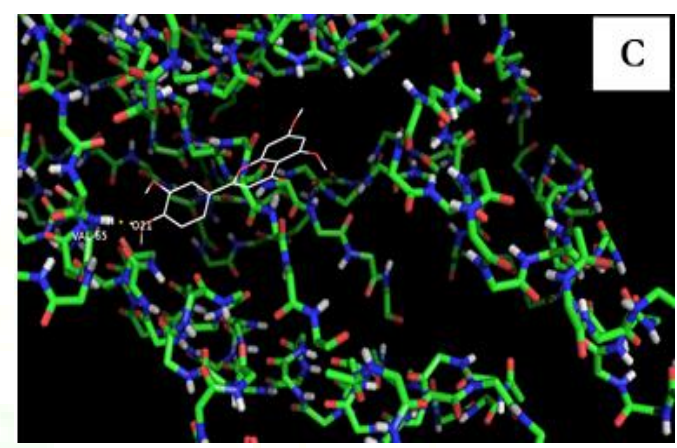

Figure 1: Docked poses: Binding mode of top ranked docked poses into InhA binding cavity with AutoDock/Vina. A. Quercetin, B. Nimocinolide, C. Luteolin

Compound dianabol of neem polyphenol showed a binding affinity of $-7.8 \mathrm{kcal} / \mathrm{mol}$ and formed one hydrogen bond between $\mathrm{O}_{12}$ and $\mathrm{O}$ of Gly14. Whereas, nimocinolide having a binding affinity of $8.0 \mathrm{kcal} / \mathrm{mol}$ formed three hydrogen bonds: one between $\mathrm{O}_{23}$ and $\mathrm{O}$ of Ala154, secondly between $\mathrm{O}_{41}$ and $\mathrm{H}$ of Gln 214 and finally between $\mathrm{H}_{48}$ and O of Asp 150 (Fig. 1B).

Meanwhile luteolin from ginkgobiloba had a binding affinity of $-8.6 \mathrm{kcal} / \mathrm{mol}$ which was the best of the selected top 8 candidates. This compound shared a hydrogen bond between $\mathrm{O}_{21}$ and $\mathrm{H}$ of Val65 as seen in Fig. 1C. The binding affinity of kaempferol was $-8.5 \mathrm{kcal} / \mathrm{mol}$ and bonded to the protein with two hydrogen bonds: one between $\mathrm{O}_{19}$ and $\mathrm{H}$ of $\mathrm{Val} 65$ while the other between $\mathrm{H}_{30}$ and $\mathrm{O}$ of Gly96. Likewise, quercetin and catechin from ginkgobiloba showed a binding affinity of $8.2 \mathrm{kcal} / \mathrm{mol}$ and $-8.0 \mathrm{kcal} / \mathrm{mol}$ respectively. Both of them form only one hydrogen bond each with the target protein. Quercetin forms hydrogen bond between $\mathrm{H}_{31}$ and $\mathrm{O}$ of Ile194 while catechin forms three hydrogen bonds. One bond is between $\mathrm{H}_{29}$ and side chain of Asp64 while the other two are between $\mathrm{H}_{35}$ and $\mathrm{O}, \mathrm{O}_{21}$ and $\mathrm{H}$ of Gly96. The compound quercetin found both in tea as well as in Ginkgo biloba plants which had the highest drug likeness score of 0.93 showed promising binding affinities with Mtb InhA. This compound was also found to target enoyl-ACP reductases of $P$. falciparum (Brown et al., 2007). 
It is worth mentioning here that residues Gly14, Ser20, Asp64, Val65, Ile95, Lys165 and Ile194 of the $M t b$ InhA which interacted with co-crystallised ligand and is within the NADH binding site of the activated complex as seen in Fig. 2. This clearly indicates that the selected ligands for the study from plant compounds essentially bound to the binding region of the $M t b \operatorname{InhA}$ protein in the presence of triclosan, the substrate binding loop (residues 195210 in InhA) of InhA structures are disordered. Although the IC50 values improved InhA structure with 5-pentyl-2-phenoxyphenol and derivatives of 8PP triclosan, they persist to be rapidly reversible inhibitors (Sullivan et al., 2006). Nevertheless, it was significant that all the top 8 selected candidates exhibited high favorable scores than the cocrystallised ligand receptor complex.

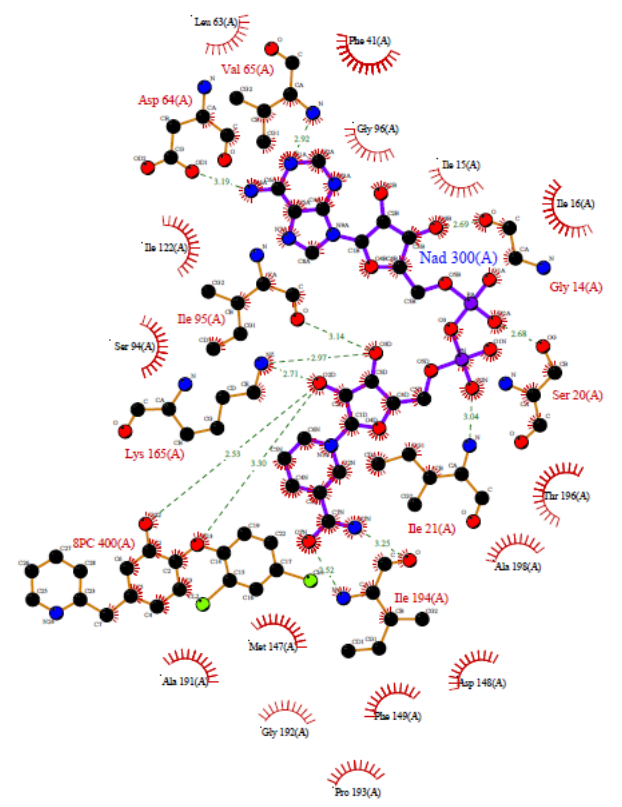

Figure 2: Docked conformation of InhA with top ranked ligands showing the interaction with the crucial residues in the active site cleft using AutoDock/Vina

\section{Conclusion}

Several potential inhibitory compounds of $M t b$ are identified in this study. These compounds bound to the NAD active binding site of InhA with higher affinities than the known co-crystallised ligands. Thus these could be a good natural alternative to the first line drugs like isoniazid and ethionamide in controlling MDR-TB. All of these compounds bound to the respective binding sites in the binding pocket. Therefore, these ginkgobiloba, tea and neem compounds can be purified and further used for developing efficient anti-TB drug. In vivo experiments with these compounds could be performed in mice to check its effects and interactions for developing an applied drug for TB. Our study suggests a basis for the design and development of natural novel drugs against TB.

\section{References}

1. Anonymous, World Health Organisation: Global tuberculosis control: WHO report (2013).

2. Banerjee, A., Dubnau, E., Quemard, A., Balasubramanian, V., Um, K.S., Wilson, T., Collins, D., de Lisle, G., Jacobs Jr, W.R., InhA, a gene encoding a target for isoniazid and ethionamide in Mycobacterium tuberculosis. Science. 263, (1994): 227230.

3. Brown, A.K., Papaemmanouil, A., Bhowruth, V., Bhatt, A., Dover, L.G., Besra, G.S., Flavonoid inhibitors as novel antimycobacterial agents targeting Rvo636, a putative dehydratase enzyme involved in Mycobacterium tuberculosis fatty acid synthase II. Microbiology. 153, (2007): 3314-3322.

4. Dessen, A., Quemard, A., Blanchard, J.S., Jacobs, W.R., Sacchettini, J.C., Crystal structure and function of the isoniazid target of in Mycobacterium tuberculosis. Science. 267, (1995): 1638-1641.

5. Gbotolorun, S.C., Osinubi, A.A., Noronha, C.C., Okanlawon, A.O., Antifertility potential of Neem flower extract on adult female SpragueDawley rats. Afr Health Sci. 8, (2008): 168-173.

6. Hasan, S., Daugelat, S., Rao, P.S.S., Schreiber, M., Prioritizing Genomic Drug Targets in Pathogens: Application to Mycobacterium tuberculosis. PLoS Comput Biol. 2.6, (2006): e61. doi: 10.1371/journal.pcbi.0020061.

7. Kakati, D., Mahanta, S., Tanti, B., In-silico comparative structural modeling of carbonic anhydrase of the marine diatom Thalassiosira pseudonana. Journal of Research in Bioinformatics. 1, (2012): 9-15.

8. Lalitha, P., Sivakamasundari, S., Calculation of Molecular lipophilicity and drug likeness for few heterocycles. Orintal J. of Chemistry. 26, (2010): 135141.

9. Laurie, A.T., Jackson, R.M., Q-SiteFinder: an energybased method for the prediction of protein-ligand binding sites. Bioinformatics. 21.9, (2005): 1908-16.

10. Lim, Y.M., Flavin, M.T., Cassidy, C.S., Mar, A., Chen, F.C., Biflavonoids as antituberculosis agents. Bioorg Med Chem Lett. 11, (2001): 2101-2104.

11. Lin, Y.M., Zhou, Y., Flavin, M.T., Zhou, L.M., Nie, W., Chen, F.C., Chalcones and flavonoids as antituberculosis agents. Bioorg Med Chem. 10, (2002): 2795-2802.

12. Lipinski, C.A., Drug- like properties and the causes of poor solubility and poor permeability. $J$ Pharmacol Toxicol Methods. 44, (2000): 235 - 249.

13. Morris, G.M., Huey, R., Olson, A.J., Using AutoDock for ligand-receptor docking. Curr Protoc Bioinformatics. Chapter 8(Unit 8), (2008): 14. 
14. Nunn, P., Williams, B., Floyd, K., Dye, C., Elzinga, G., Raviglione, M., Tuberculosis control in the era of HIV. Nat Rev Immunol. 5, (2005): 819-826.

15. Pedretti, A., Villa, L., Vistoli, G., VEGA - An open platform to develop chemo-bioinformaitcs applications, using plug-in architechture and script programming. Journal of Molecular Aided Drug Designing. 18, (2004): 167-173.

16. Pettersen, E.F., Goddard, T.D., Huang, C.C., Couch, G.S., Greenbaltt, D.N., Meng, E.C., Ferrin, T.E., UCSF Chimera- A visualization system for exploratory research and analysis. J. Comput. Chem. 25.13, (2004): 1605- 1612.

17. Quemard, A., Sacchettini, J.C., Dessen, A., Vilcheze, C., Bittman, R., Jacobs, W.R., Blanchard, J.S., Enzymatic characterization of the target for isoniazid in Mycobacterium tuberculosis. Biochemistry 34, (1995): 8235-8241.

18. Raman, K., Yeturu, K., Chandra, N., Target TB: A target identification pipeline for Mycobacterium tuberculosis through an interactome, reactome and genome-scale structural analysis. BMC Systems Biology. 2, (2008):109.

19. Sadowski, J., Gasteiger, J., Klebe G., Comparision of Automatic Three-Dimensional Model Builders Using 639 X-ray Structures. J. Chem. Inf. Comput. Sci. 34, (1994): 1000-1008.

20. Schroeder, E.K., de Souza, N., Santos, D.S., Blanchard, J.S., Basso, L.A., Drugs that inhibit mycolic acid biosynthesis in Mycobacterium tuberculosis. Curr Pharm Biotechnol. 3, (2002): 197225.

21. Schumacher, M., Cerella, C., Reuter, S., Dicato, M., Diederic, M., Anti-inflammatory, pro-apoptotic, and anti-proliferative effects of a methanolic neem (Azadirachta indica) leaf extract are mediated via modulation of the nuclear factor- $x \mathrm{~B}$ pathway. Genes Nutr. 6, (2011):149-160.

22. Sharma, D.K., Pharmacological properties of flavanoids including flavonolignans-Integration of petrocrops with drug development from plants. Journal of Scientific and Industrial Research. 65, (2006): 477-484.

23. Sullivan, T.J., Truglio, J.J., Boyne, M.E., Novichenok, P., Zhang, X., Stratton, C.F., Li, H.J., Kaur, T., Amin, A., Johnson, F., Slayden, R.A., Kisker, C., Tonge, P.J., High affinity InhA inhibitors with activity against drug-resistant strains of Mycobacterium tuberculosis. ACS Chem Biol. 1, (2006): 43-53.

24. Trott, O., Olson, A.J., Auto Dock Vina: improving the speed and accuracy of docking with a new scoring function, efficient optimization, and multithreading. J Comput Chem. 31.2, (2010): 455-461.

25. Vilchèze, C., Wang, F., Arai, M., Hazbón, M.H., Colangeli, R., Kremer, L., Weisbrod, T.R., Alland, D., Sacchettini, J.C., Jacobs Jr, W.R., Transfer of a point mutation in Mycobacterium tuberculosis inhA resolves the target of isoniazid. Nat Med. 12, (2006): 1027-1029.

26. Wang, H., Ng, T.B., Ginkbilobin, a novel antifungal protein from Ginkgo biloba seeds with sequence similarity to embryo-abundant protein. Biochem Biophys Res Commun. 279, (2000): 407-11.

\section{Cite this article as:}

Saurov Mahanta, Purvita Chowdhury, Shamsun Nahar, Bhaben Tanti, P.J. Handique. Discovery of natural inhibitors targeting 2 - trans enoyl acyl carrier protein reductase in Mycobacterium tuberculosis by structure based drug designing. Annals of Plant Sciences 5.9 (2016): 1417-1423.

DOI: http://dx.doi.org/10.21746/aps.2016.09.002

Source of support: Nil.

Conflict of interest: None Declared 